\title{
Reduced second Zagreb index of product graphs
}

\author{
N. De \\ Department of Basic Sciences and Humanities (Mathematics), \\ Calcutta Institute of Engineering and Management, Kolkata, India \\ de.nilanjan@rediffmail.com
}

DOI 10.17586/2220-8054-2020-11-2-131-137

\begin{abstract}
The reduced second Zagreb index of a graph $G$ is defined as $R M_{2}(G)=\sum_{u v \in E(G)}\left(d_{G}(u)-1\right)\left(d_{G}(v)-1\right)$, where $d_{G}(v)$ denotes the degree of the vertex $v$ of graph $G$. Recently Furtula et al. (Furtula B., Gutman I., Ediz S. Discrete Appl. Math., 2014) characterized the maximum trees with respect to reduced second Zagreb index. The aim of this paper is to compute reduced second Zagreb index of the Cartesian product of $k(\geq 2)$ number of graphs and hence as a consequence the reduced second Zagreb index of some special graphs applicable in various real world problems are computed. Topological properties of different nanomaterials like nanotube, nanotorus etc. are studied here graphically in terms of the aforesaid aforementioned index.
\end{abstract}

Keywords: Reduced second Zagreb index, cartesian product of graphs, nanotube, nanotorus, Hamming graphs, Ladder graphs, Rook's graph.

Received: 15 January 2020

Revised: 5 March 2020

\section{Introduction}

Let $G$ be a simple connected graph with vertex set $V(G)$ and edge set $E(G)$. For a graph $G$, let $d_{G}(v)$ denote the degree of a vertex $v$ in $G$, that is, the number of vertices adjacent with $v$. Throughout this article, we consider chemical graph [1,2]. By chemical graph we mean a simple connected graph where vertices and edges are supposed to be atoms and chemical bonds between them respectively.

Topological indices, also called molecular structure descriptors are used in theoretical chemistry for design of chemical compounds with given physico-chemical properties and also to model chemical biological and pharmacological properties of the molecules. A topological index is a real number and it does not depend on the labelling of a graph and must be a structural invariant. The first topological index, Wiener index, was published in 1947 [3]. Due to the importance of topological indices in chemical research, lots of topological indices are developed in the chemical graph theory. Degree based topological indices are one of them that is applicable in quantitative structure property relationship and quantitative structure activity relationship [4,5]. Among them Zagreb indices are most popular indices. First and second Zagreb indices are introduced by Gutman and Trinajestić [6], defined as follows:

$$
\begin{aligned}
& M_{1}(G)=\sum_{u \in V(G)} d_{G}(u)^{2}, \\
& M_{2}(G)=\sum_{u v \in E(G)} d_{G}(u) d_{G}(v) .
\end{aligned}
$$

These graph invariants were proposed to measure the branching of carbon-atom skeleton [7]. For detail discussion on these indices, see [8-14]. Furtula et al. [15] proposed the reduced second Zagreb index of a graph $G$ to study the difference between $M_{1}$ and $M_{2}$, which is defined as follows:

$$
R M_{2}(G)=\sum_{u v \in E(G)}\left(d_{G}(u)-1\right)\left(d_{G}(v)-1\right) .
$$

The graphs having maximum and minimum reduced second Zagreb index in the class of cyclic graphs with cut edges are studied in [16]. Mahanta et al. [17] obtained Reduced the reduced second Zagreb index of four new graph operations based on tensor product. Several useful composite graphs can be obtained by operations of different graphs. The Cartesian product is one of that the operations and which is considered in the present work.

The Cartesian product $G_{1} \otimes G_{2}$ of graphs $G_{1}$ and $G_{2}$ has the vertex set $V(G) \times V\left(G_{2}\right)$ and $(a, x)(b, y)$ is an edge of $G_{1} \otimes G_{2}$ if $a=b$ and $x y \in E(H)$ or $a b \in E(G)$ and $x=y$. Using the Cartesian product of two graphs, the structure of several nanomaterials can be designed such as $C_{4}$-nanotube, $C_{4}$-nanotorus, multi-walled nanotorus etc. Carbon nanotube has diverse applications in cancer treatment, cardiac autonomic regulation, tissue regeneration etc. As topological index can predict different physico-chemical properties, it is worthy to compute that for different 
structures of real world application. First and second Zagreb indices of the Cartesian product graph is presented in [18]. The PI index of the Cartesian product of bipartite graphs is computed in [19]. Klavzar et al. [20] computed the Szeged index of Cartesian product graph. In [21], the Wiener index of Cartesian product graphs are studied. The present author [22-24] studied F-index, F-coindex and reformulated first Zagreb index for Cartesian product graphs. The goal of this work is to obtain the reduced second Zagreb index of Cartesian product graphs. Using that results, $R M_{2}$ index of some chemical graphs is also derived.

\section{Main results}

Let $G_{i}(i=1,2, \ldots, k)$ be a connected graph with vertex set $V\left(G_{i}\right)$ and $E\left(G_{i}\right)$, so that, $\left|V\left(G_{i}\right)\right|=n_{i}$ and $\left|E\left(G_{i}\right)\right|=m_{i}$. In this section, we derive the reduced second Zagreb index of Cartesian product of $k$-number of connected graphs $G_{1}, G_{2}, \ldots, G_{k}$. To do this first we prove the result for two connected graphs $G_{1}$ and $G_{2}$.

Lemma 1. [18] Let $G_{1}$ and $G_{2}$ be two connected graphs, then:

$$
\begin{aligned}
(i)\left|V\left(G_{1} \otimes G_{2}\right)\right| & =\left|V\left(G_{1}\right)\right| \times\left|V\left(G_{2}\right)\right|, \\
(i i)\left|E\left(G_{1} \otimes G_{2}\right)\right| & =\left|E\left(G_{1}\right)\right|\left|V\left(G_{2}\right)\right|+\left|E\left(G_{2}\right)\right|\left|V\left(G_{1}\right)\right|, \\
(\text { iii }) d_{G_{1} \otimes G_{2}}(a, b) & =d_{G_{1}}(a)+d_{G_{2}}(b) .
\end{aligned}
$$

Lemma 2. [18] Let $G_{1}, G_{2}, \ldots ., G_{k}$ be k-number of graphs, then

$$
M_{1}\left(\bigotimes_{i=1}^{k} G_{i}\right)=n \sum_{i=1}^{k} \frac{M_{1}\left(G_{i}\right)}{n_{i}}+4 n \sum_{i \neq j, i, j=1}^{k} \frac{m_{i} m_{j}}{n_{i} n_{j}} .
$$

Lemma 3. [18] Let $G_{1}, G_{2}, \ldots$, $G_{k}$ be k-number of graphs, then

$$
\begin{aligned}
(i)\left|V\left(\bigotimes_{i=1}^{k} G_{i}\right)\right| & =\prod_{i=1}^{k} n_{i}, \\
(i i)\left|E\left(\bigotimes_{i=1}^{k} G_{i}\right)\right| & =\prod_{i=1}^{k} n_{i} \sum_{i=1}^{k} \frac{m_{i}}{n_{i}}
\end{aligned}
$$

Theorem 1. Let $G_{1}$ and $G_{2}$ be two connected graphs with $n_{1}$ and $n_{2}$ number of vertices and, $m_{1}$ and $m_{2}$ number of edges respectively, then:

$$
R M_{2}\left(G_{1} \otimes G_{2}\right)=n_{1} R M_{2}\left(G_{2}\right)+n_{2} R M_{2}\left(G_{1}\right)+3 m_{1} M_{1}\left(G_{2}\right)+3 m_{2} M_{1}\left(G_{1}\right)-8 m_{1} m_{2} .
$$

Proof. From definition of Cartesian product of two graphs, we have:

$$
\begin{aligned}
R M_{2}\left(G_{1} \otimes G_{2}\right)= & \sum_{(a, b)(c, d) \in E\left(G_{1} \otimes G_{2}\right)}\left(d_{G_{1}}(a, b)-1\right)\left(d_{G_{2}}(c, d)-1\right) \\
= & \sum_{u \in V\left(G_{1}\right)} \sum_{b d \in E\left(G_{2}\right)}\left(d_{G_{1}}(u)+d_{G_{2}}(b)-1\right)\left(d_{G_{1}}(u)+d_{G_{2}}(d)-1\right) \\
& +\sum_{v \in V\left(G_{2}\right)} \sum_{a c \in E\left(G_{1}\right)}\left(d_{G_{1}}(a)+d_{G_{2}}(v)-1\right)\left(d_{G_{1}}(c)+d_{G_{2}}(v)-1\right) \\
= & C_{1}+C_{2},(\text { Say }) .
\end{aligned}
$$

Where,

$$
\begin{aligned}
C_{1}= & \sum_{u \in V\left(G_{1}\right)} \sum_{b d \in E\left(G_{2}\right)}\left(d_{G_{1}}(u)+d_{G_{2}}(b)-1\right)\left(d_{G_{1}}(u)+d_{G_{2}}(d)-1\right) \\
= & \sum_{u \in V\left(G_{1}\right)} \sum_{b d \in E\left(G_{2}\right)}\left(d_{G_{2}}(b)-1\right)\left(d_{G_{2}}(d)-1\right)+\sum_{b d \in E\left(G_{2}\right)} \sum_{u \in V\left(G_{1}\right)} d_{G_{1}}(u)^{2} \\
& +\sum_{b d \in E\left(G_{2}\right)} \sum_{u \in V\left(G_{1}\right)} d_{G_{1}}(u)\left(d_{G_{2}}(b)+d_{G_{2}}(d)-2\right) \\
= & n_{1} R M_{2}\left(G_{2}\right)+m_{2} M_{1}\left(G_{1}\right)+2 m_{1} M_{1}\left(G_{2}\right)-4 m_{1} m_{2} .
\end{aligned}
$$


Similarly,

$$
\begin{aligned}
C_{2}= & \sum_{v \in V\left(G_{2}\right)} \sum_{a c \in E\left(G_{1}\right)}\left(d_{G_{1}}(a)+d_{G_{2}}(v)-1\right)\left(d_{G_{1}}(c)+d_{G_{2}}(v)-1\right) \\
= & \sum_{v \in V\left(G_{2}\right)} \sum_{a c \in E\left(G_{1}\right)}\left(d_{G_{1}}(a)-1\right)\left(d_{G_{1}}(c)-1\right)+\sum_{a c \in E\left(G_{1}\right)} \sum_{v \in V\left(G_{2}\right)} d_{G_{1}}(v)^{2} \\
& +\sum_{a c \in E\left(G_{1}\right)}\left(d_{G_{1}}(a)+d_{G_{1}}(c)-2\right) \sum_{v \in V\left(G_{2}\right)} d_{G_{1}}(v) \\
= & n_{2} R M_{2}\left(G_{1}\right)+m_{1} M_{1}\left(G_{2}\right)+2 m_{2} M_{1}\left(G_{1}\right)-4 m_{1} m_{2} .
\end{aligned}
$$

Therefore, combining the contributions of $C_{1}$ and $C_{2}$ we get the desired results.

In the following, we calculate reduced second Zagreb index of the Cartesian product of $k$-number of graphs $G_{i}$ for $i=1,2, \ldots, k$.

Theorem 2. Let $G_{1}, G_{2}, \ldots . ., G_{k}$ be k-number of connected graphs, then

$$
\begin{aligned}
R M_{2}\left(\bigotimes_{i=1}^{k} G_{i}\right)= & n \sum_{i=1}^{k} \frac{R M_{2}\left(G_{i}\right)}{n_{i}}+3 \sum_{i=1}^{k}\left(\frac{m}{n_{i}}-\frac{n m_{i}}{n_{i}^{2}}\right) M_{1}\left(G_{i}\right) \\
& +4 n \sum_{p, q, r=1, p \neq q \neq r} \frac{m_{p} m_{q} m_{r}}{n_{p} n_{q} n_{r}}-4 n \sum_{i, j=1, i \neq j} \frac{m_{i} m_{j}}{n_{i} n_{j}}
\end{aligned}
$$

Where, $n=\left|V\left(\bigotimes_{i=1}^{k} G_{i}\right)\right|$ and $m=\left|E\left(\bigotimes_{i=1}^{k} G_{i}\right)\right|$.

Proof. Let us assume that, $n^{\prime}=\left|V\left(\bigotimes_{i=1}^{k-1} G_{i}\right)\right|$ and $m^{\prime}=\left|E\left(\bigotimes_{i=1}^{k-1} G_{i}\right)\right|$ so that, $n^{\prime}=\frac{n}{n_{k}}$ and $m^{\prime}=\frac{m n_{k}-n m_{k}}{n_{k}^{2}}$. Now by Theorem 1 and an inductive argument, we can have

$$
\begin{aligned}
R M_{2}\left(\bigotimes_{i=1}^{k} G_{i}\right)= & R M_{2}\left(\bigotimes_{i=1}^{k-1} G_{i} \otimes G_{k}\right) \\
= & n_{k} R M_{2}\left(\bigotimes_{i=1}^{k-1} G_{i}\right)+\left|V\left(\bigotimes_{i=1}^{k-1} G_{i}\right)\right| R M_{2}\left(G_{k}\right)+3 m_{k} M_{1}\left(\bigotimes_{i=1}^{k-1} G_{i}\right) \\
& +3\left|E\left(\bigotimes_{i=1}^{k-1} G_{i}\right)\right| M_{1}\left(G_{k}\right)-8\left|E\left(\bigotimes_{i=1}^{k-1} G_{i}\right)\right|\left|E\left(G_{k}\right)\right|
\end{aligned}
$$

Using Lemmas 1, 2, and 3 on the above result, we obtain:

$$
\begin{aligned}
R M_{2}\left(\bigotimes_{i=1}^{k} G_{i}\right)= & n_{k}\left[n^{\prime} \sum_{i=1}^{k-1} \frac{R M_{2}\left(G_{i}\right)}{n_{i}}+3 \sum_{i=1}^{k-1}\left(\frac{m^{\prime}}{n_{i}}-\frac{n^{\prime} m_{i}}{n_{i}^{2}}\right) M_{1}\left(G_{i}\right)\right. \\
& \left.+4 n^{\prime} \sum_{p, q, r=1, p \neq q \neq r}^{k-1} \frac{m_{p} m_{q} m_{r}}{n_{p} n_{q} n_{r}}-4 n^{\prime} \sum_{i, j=1}^{k-1} \frac{m_{i} m_{j}}{n_{i} n_{j}}\right]+n^{\prime} R M_{2}\left(G_{k}\right) \\
& +3 m_{k}\left[n^{\prime} \sum_{i=1}^{k-1} \frac{M_{1}\left(G_{i}\right)}{n_{i}}+4 n^{\prime} \sum_{i, j=1}^{k-1} \frac{m_{i} m_{j}}{n_{i} n_{j}}\right]+3 n^{\prime} \sum_{i=1}^{k-1}\left(\frac{m_{i}}{n_{i}} M_{1}\left(G_{k}\right)\right. \\
& -8 m_{k} n^{\prime} \sum_{i=1}^{k} \frac{m_{i}}{n_{i}}
\end{aligned}
$$


After arranging the terms, we have:

$$
\begin{aligned}
R M_{2}\left(\bigotimes_{i=1}^{k} G_{i}\right)= & {\left[n_{k} n^{\prime} \sum_{i=1}^{k-1} \frac{R M_{2}\left(G_{i}\right)}{n_{i}}+n^{\prime} R M_{2}\left(G_{k}\right)\right]+\left[3 n_{k} \sum_{i=1}^{k-1}\left(\frac{m^{\prime}}{n_{i}}-\frac{n^{\prime} m_{i}}{n_{i}^{2}}\right) M_{1}\left(G_{i}\right)\right.} \\
& +3 m_{k} n^{\prime} \sum_{i=1}^{k-1} \frac{M_{1}\left(G_{i}\right)}{n_{i}}+3 n^{\prime} \sum_{i=1}^{k-1}\left(\frac{m_{i}}{n_{i}} M_{1}\left(G_{k}\right)\right] \\
& +\left[4 n_{k} n^{\prime} \sum_{p, q, r=1, p \neq q \neq r}^{k-1} \frac{m_{p} m_{q} m_{r}}{n_{p} n_{q} n_{r}}+12 n^{\prime} m_{k} \sum_{i, j=1}^{k-1} \frac{m_{i} m_{j}}{n_{i} n_{j}}\right] \\
& -\left[4 n \sum_{i, j=1}^{k-1} \frac{m_{i} m_{j}}{n_{i} n_{j}}+8 m_{k} n^{\prime} \sum_{i=1}^{k} \frac{m_{i}}{n_{i}}\right]
\end{aligned}
$$

After some calculations, we get:

$$
\begin{aligned}
R M_{2}\left(\bigotimes_{i=1}^{k} G_{i}\right)= & {\left[n \sum_{i=1}^{k-1} \frac{R M_{2}\left(G_{i}\right)}{n_{i}}+n \frac{R M_{2}\left(G_{k}\right)}{n_{k}}\right]+\left[3 \sum _ { i = 1 } ^ { k - 1 } \left(\frac{m^{\prime} n_{k}+m_{k} n^{\prime}}{n_{i}}\right.\right.} \\
& \left.\left.-\frac{n^{\prime} n_{k} m_{i}}{n_{i}^{2}}\right) M_{1}\left(G_{i}\right)+3\left(\frac{m n_{k}-n m_{k}}{n_{k}^{2}}\right) M_{1}\left(G_{k}\right)\right] \\
+ & {\left[4 n \sum_{p, q, r=1, p \neq q \neq r}^{k-1} \frac{m_{p} m_{q} m_{r}}{n_{p} n_{q} n_{r}}+12 n \frac{m_{k}}{n_{k}} \sum_{i, j=1, i \neq j}^{k-1} \frac{m_{i} m_{j}}{n_{i} n_{j}}\right] } \\
& -\left[4 n \sum_{i, j=1}^{k-1} \frac{m_{i} m_{j}}{n_{i} n_{j}}+8 n \frac{m_{k}}{n_{k}} \sum_{i=1}^{k} \frac{m_{i}}{n_{i}}\right] \\
= & n \sum_{i=1}^{k} \frac{R M_{2}\left(G_{i}\right)}{n_{i}}+3 \sum_{i=1}^{k}\left(\frac{m}{n_{i}}-\frac{n m_{i}}{n_{i}^{2}}\right) M_{1}\left(G_{i}\right) \\
& \left.+4 n \sum_{p, q, r=1, p \neq q \neq r} \frac{m_{p} m_{q} m_{r}}{n_{p} n_{q} n_{r}}-4 n \sum_{i, j=1}^{k} \frac{m_{i} m_{j}}{n_{i} n_{j}}\right]
\end{aligned}
$$

which is the desired result.

$$
\text { If } G_{1}=G_{2}=\ldots \ldots .=G_{k}=G \text { then } \bigotimes_{i=1}^{k} G_{i}=G^{k} \text {. Hence from the above theorem, the following corollary }
$$
follows:

Corollary 1. If $G$ be connected graph, then

$$
\begin{aligned}
R M_{2}\left(G^{k}\right)= & k|V(G)|^{k-3}\left[|V(G)|^{2} R M_{2}(G)+3(k-1)|V(G)||E(G)| M_{1}(G)\right. \\
& \left.+4(k-1)(k-2)|E(G)|^{3}-4(k-1)|V(G)||E(G)|^{2}\right] .
\end{aligned}
$$

\section{Applications}

Using various unary and binary graph operations on different elementary graphs, such as the path graph, cycle graph, complete graph etc, we can obtain several significant composite graphs having excellent usage in modern science and technology. The Cartesian product is one of that binary operations capable to construct different special structure. Using Cartesian product of two graphs, one can get ladder graph, $C_{4}$-nanotube and nanotorus, rectangular grid, rook's graph, hamming graph etc. The Hamming graphs are interesting in connection with error-correcting codes and association schemes. The rook's graph represents all legal moves of rook on the chess board. So it is worth to investigate investigating topological indices for the above structures. In this section, the reduced second Zagreb index of aforesaid graphs is derived. For path, cycle, and complete graph of $n$ vertices, the notations $P_{n}, C_{n}$, and $K_{n}$ are used.

Example 1. The Ladder graph $L_{n}$ (Fig. 1) is the Cartesian product of $P_{2}$ and $P_{n+1}$, made by $n$ sequences and $(2 n+2)$ vertices. So, using theorem 1, the reduced second Zagreb index of $L_{n}$ is given by

$$
R M_{2}\left(L_{n}\right)=12 n-10 \text {. }
$$




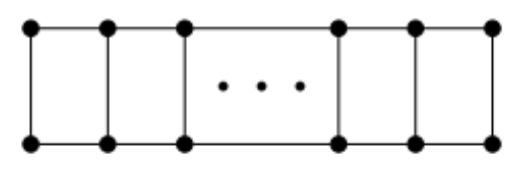

FIG. 1. The ladder graph $L_{n}$

Example 2. The Cartesian product of $P_{n}(n \geq 2)$ and $C_{m}(m \geq 2)$ is a $C_{4}$-nanotube $T U C_{4}(m, n)$, whose reduced second Zagreb index can be calculated using theorem 1 as follows:

$$
R M_{2}\left(P_{n} \otimes C_{m}\right)=18 m n-25 m .
$$

Example 3. The Cartesian product of $C_{n}(n \geq 3)$ and $C_{m}(m \geq 3)$ is a $C_{4}$-nanotorus $T C_{4}(m, n)$, whose reduced second Zagreb index is calculated from Theorem 1 as follows:

$$
R M_{2}\left(C_{n} \otimes C_{m}\right)=18 m n \text {. }
$$

Example 4. The rectangular grid (Fig.2) is the Cartesian product of the $P_{n}(n \geq 2)$ and $P_{m}(m \geq 2)$. So, using Theorem 1, its reduced second Zagreb index is given by

$$
R M_{2}\left(P_{n} \otimes P_{m}\right)=18 m n-25 m-25 n+28 .
$$

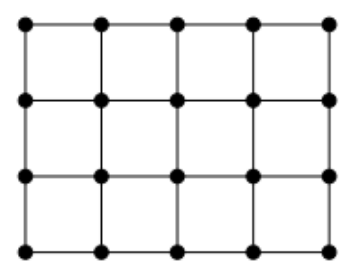

FIG. 2. The grid graph $P_{5} \otimes P_{4}$

Example 5. The Cartesian product of $K_{n}$ and $K_{m}$ yields the rook's graph (Fig. 3). So, using Theorem 1, its reduced second Zagreb index is given by

$$
R M_{2}\left(K_{m} \otimes K_{n}\right)=\frac{m n}{2}\left[(m+n)^{3}-8 m^{2}-8 n^{2}-16 m n+21 m+21 n-14\right] .
$$

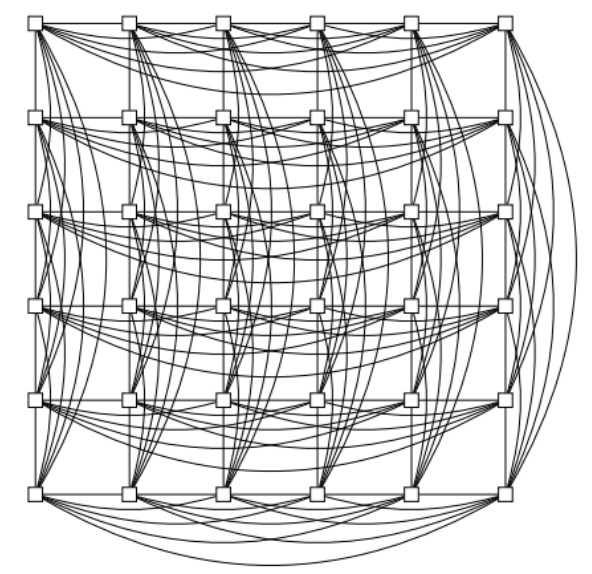

FIG. 3. The rook's graph $K_{6} \otimes K_{6}$ 
Example 6. The graph $G=\bigotimes_{i=1}^{N} K_{n_{i}}$ is known as a Hamming graph and is denoted by $H_{n_{1}, n_{2}, \ldots, n_{N}}$. so, applying previous theorem to compute reduced second Zagreb index of a Hamming graph as follows:

$$
\begin{aligned}
R M_{2}\left(H_{n_{1}, n_{2}, \ldots, n_{N}}\right) & =R M_{2}\left(\bigotimes_{i=1}^{N} K_{n_{i}}\right) \\
& =\frac{1}{2} \prod_{i=1}^{N} n_{i} \sum_{i=1}^{N}\left(n_{i}-1\right)\left[\sum_{i=1}^{N}\left(n_{i}-1\right)-1\right]^{2} .
\end{aligned}
$$

If $n_{1}=n_{2}=\ldots \ldots .=n_{N}=2$, then the graph $G$ is known as a hypercube of dimension $N$ and denoted by $Q_{n}$ (Fig. 4). Thus from above, we directly get

$$
R M_{2}\left(Q_{n}\right)=\frac{1}{2} N 2^{N}(N-1)^{2} .
$$

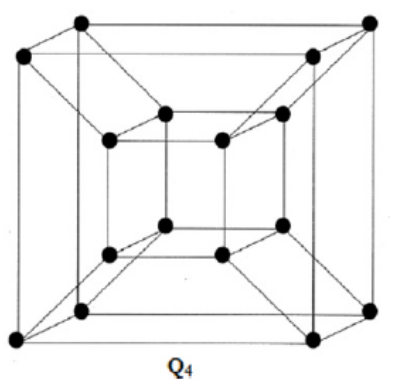

FIG. 4. Example of Hypercube

Example 7. The reduced second Zagreb index of the torus $C_{n_{1}} \otimes C_{n_{2}} \otimes \ldots \ldots \otimes C_{n_{k}}$ is given by

$$
R M_{2}\left(C_{n_{1}} \otimes C_{n_{2}} \otimes \ldots \ldots \otimes C_{n_{k}}\right)=k(2 k-1)^{2} \prod_{i=1}^{k} n_{i} .
$$

Example 8. Let $T=T[p, q]$ be the molecular graph of a nanotorus (Fig. 5). Then $|V(T)|=p q,|E(T)|=\frac{9 p q}{2}$, $M_{1}(T)=9 p q$. We consider a q-multi-walled nanotorus $G_{n}=P_{n} \otimes T$. It is easy to find that $R M_{2}\left(P_{n}\right)=n-3$, $R M_{2}(T)=6 p q$, and $M_{1}\left(P_{n}\right)=4 n-6$. Thus from theorem 1 , we have the following result.

$$
R M_{2}\left(G_{n}\right)=5 p q(8 n-9) .
$$

We have plotted the result in Fig. 6

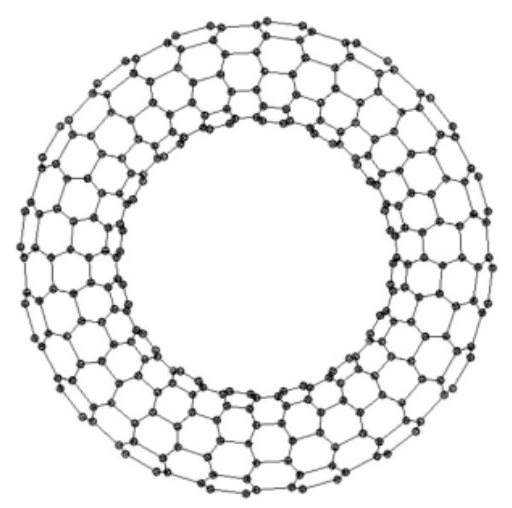

FIG. 5. The graph of a nanotorus 


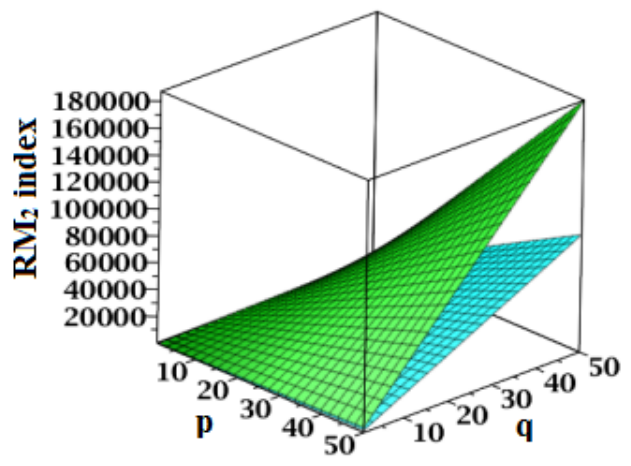

FIG. 6. Plotting of the $R M_{2}$ index for $G_{n}, n=2,3$. Cyan and green colors are used for $n=2,3$ respectively

\section{Conclusion}

In this article, reduced second Zagreb index of Cartesian product graph is studied. Firstly $R M_{2}$ index is obtained for the product of two graphs and then the general case is considered. Applying that results, $R M_{2}$ index is investigated for some special structures. As future work, some other graph operations like composition, tensor product, corona product, strong product, splice, link etc. can be discussed in terms of the reduced second Zagreb index.

\section{References}

[1] Trinajstić N. Chemical Graph Theory. CRC Press, Boca Raton, 1993.

[2] Gutman I., Polansky O.E. Mathematical Concepts in Organic Chemistry. Springer, Berlin, 1986.

[3] Wiener H. Structural determination of paraffin boiling points. J. Am. Chem. Soc., 1947, 69, P. 7-20.

[4] Randić M. Quantitative Structure - Property Relationship: boiling points and planar benzenoids. New. J. Chem., 1996, 20(10), P. 1001-1009.

[5] Hawkins D.M., Basak S.C., Mills D. Assessing Model Fit by Cross Validation. J. Chem. Inf. Comput. Sci., 2003, 43, P. 579-586.

[6] Gutman I, Trinajstić N. Graph theory and molecular orbitals. Total $\pi$-electron energy of alternate hydrocarbons. Chem. Phys. Lett., 1972, 17, P. 535-538.

[7] Gutman I, Rućić B, Trinajstić N, Wilcox C.F. Graph theory and molecular orbitals. XII. Acyclic polyenes. J. Chem. Phys., 1975, 62, P. 33993405.

[8] Das K.C., Xu K., Gutman I. On Zagreb and Harary indices. MATCH Commun. Math. Comput Chem., 2013, 70, P. 301-314.

[9] Azari M., Iranmanesh A., Gutman I. Zagreb indices of bridge and chain graphs. MATCH Commun. Math. Comput. Chem., 2013, 70, P. 921938.

[10] Hamzeh A., Reti T. An analogue of Zagreb index inequality obtained from graph ir-regularity measures. MATCH Commun. Math. Comput. Chem., 2014, 72, P. 669-683.

[11] Li S., Zhang M.J. Sharp upper bounds for the Zagreb indices of bipartite graphs with a given diameter. Appl. Math. Lett., 2011,24, P. 131-137.

[12] Fonseca C.M.D., Stevanovic D. Further properties of the second Zagreb index. MATCH Commun. Math. Comput. Chem., 2014,72 , P. 655-668.

[13] Gutman I., Das K.C. The first Zagreb index 30 years after. MATCH Commun. Math. Comput. Chem., 2004, 50, P. 83-92.

[14] Mondal S., Bhosale A., De N., Pal A. Topological properties of some nanostructures. Nanosystems: Physics, Chemistry, Mathematics, 2020, 11 (1), P. 14-24.

[15] Furtula B., Gutman I., Ediz S. On difference of Zagreb indices. Discrete Appl. Math., 2014, 178, P. 83-88.

[16] Horoldagva H., Buyantogtokh L., Dorjsembe S. Difference of Zagreb indices andreduced second Zagreb index of cyclic graphs with cut edges. MATCH Commun. Math.Comput. Chem., 2017, 78, P. 337-349.

[17] Mahanta A., Buragohain J., Bharali A. Reduced first Zagreb index, Reduced second Zagreb index and hyper-Zagreb index of four new sums based on tensor product of graphs. Global journal of engineering science and researches, 2018, 5 (11), P. 12-19.

[18] Khalifeh M.H., Azari H.Y, Ashrafi A.R. The first and second Zagreb indices of some graph operations. Discrete Appl. Math., 2009, 157, P. 804-811.

[19] Azari H.Y, Manoochehrian B, Ashrafi A.R. The PI index of product graphs. Appl. Math. Lett., 2008, 21, P. 624-627.

[20] Klavzar S., Rajapakse A., Gutman I. The Szeged and the Wiener index of graphs. Appl. Math. Lett., 1996,9 , P. 45-49.

[21] Graovac A., Pisanski T. On the Wiener index of a graph. J. Math. Chem., 1991, 8, P. 53-62.

[22] De N., Nayeem S.M.A., Pal A. F-Index of some graph operations. 2016, Discrete Math. Algorithm Appl., 8, Article ID: 1650025.

[23] De N., Nayeem S.M.A., Pal A. The F-coindex of some graph operations. 2016, Springer Plus, 5, P. $221-233$.

[24] De N., Nayeem S.M.A., Pal A. Reformulated First Zagreb Index of Some Graph Operations. Mathematics, 2015, 3(4), P. 945-960.

[25] De N., Pal A., Nayeem S.M.A. On some bounds and exact formulae for connective eccentric indices of graphs under some graph operations. Int. J. Comb., 2014, 2014, Article ID 579257. 